\title{
Use Genetic Algorithm to Sliding Mode Control of
}

\section{Active Power Filters}

\author{
Jian Wang, Jian-Ping Sheng, \\ Rui-Qi Gong, Shi-Yun Jin, \\ School of Mechatronics Engineering and Automation, \\ Shanghai University, \\ Shanghai 200072, China
}

\author{
Ye-Hua Xie \\ School of Information and Electrical Engineering, China \\ University of Mining and Technology, \\ Xuzhou 221008, China \\ E-mail: 752787576@qq.com
}

\begin{abstract}
In order to improve the defect of active power filter (APF) based on the sliding mode control (SMC), which will be always chattering at high frequencies, we proposed to take genetic algorithm into the optimization of controller parameters. And at the same time, the theoretical basis for the design of the controller is also improved. In this paper, we made a brief introduction of the working principle of threephase three-wire active power filter and current construction of the sliding mode variable structure controller, which due to a certain amount of error caused by compensating current and reference current. Though the process of the optimization based on genetic algorithm of controller parameters given in details, we got the explicit values of the controller parameters. The simulation for control and optimization of traditional PI sliding mode control shows that the sliding mode control algorithm proposed has fast response speed. It's simple, but has a better jitter-suppression and strong robustness, which is also the aim of optimization.
\end{abstract}

Keywords-sliding mode control; active power filter; genetic algorithm; parameter optimization; robustness

\section{INTRODUCTION}

With the power supply system more and more complex, a variety of power electronic devices are widely used, power quality problems have become increasingly prominent in low-voltage distribution network, to the normal operation of power equipment has brought serious impact. Power quality problems include: voltage flicker, three-phase imbalance and current distortion and other issues. To create a "green" power grid is necessary. On the one hand to make the power system safe and stable operation, on the other hand can guarantee the normal operation of electrical equipment ${ }^{[1]}$. Shunt active power filter (APF) uses PWM rectifier topology, with current source characteristics, to the grid into the compensation current, in order to reduce the power supply current distortion rate, it not only can effectively suppress the harmonic current, but can play the purpose of reactive power compensation, so that the net-side current exhibits a sine wave $^{[2]}$. Harmonic detection method and current control strategy determine the APF suppression harmonic current effect, which is good or bad. Hysteresis control mode, triangular wave control mode, deadbeat control mode, space vector modulation and so on are the commonly used current control methods. In the hysteresis control, the switching frequency with the peak voltage fluctuations will be in the corresponding fluctuations. Deadbeat control system model requires high accuracy, but poor stability under perturbation. Triangular carrier wave control is limited by the switching frequency of power electronic devices. In the high frequency range, the control performance is low. Space vector modulation control strategy can guarantee the stability of the current, but there are also control algorithm is complex, long operation time, the system real-time low shortcomings ${ }^{[3]}$.

Sliding mode control can track system state, insensitive to system parameters and disturbance, good stability and fast response. PWM rectifier by controlling the opening and closing of the bridge arm switching devices to achieve variable current, active power filter system structure is constantly changing. Based on the above characteristics, sliding mode control in the active filter can be a very good application ${ }^{[4]}$.

Based on the time domain analysis of the parallel active power filter, a sliding mode controller model is established. Furthermore, the parameters of the controller are optimized by improved genetic algorithm to improve the system design efficiency and performance. Compared with the classical double closed-loop vector control, the simulation experiment shows that the improved sliding mode controller can achieve better steady-state performance and dynamic performance.

\section{BASIC STRUCTURE AND MATHEMATICAL MODEL}

Neglecting the line impedance, the ideal parallel threephase active power filter is studied, and the mathematical model in time domain is established. The structure of the principle is shown in Figure 1.

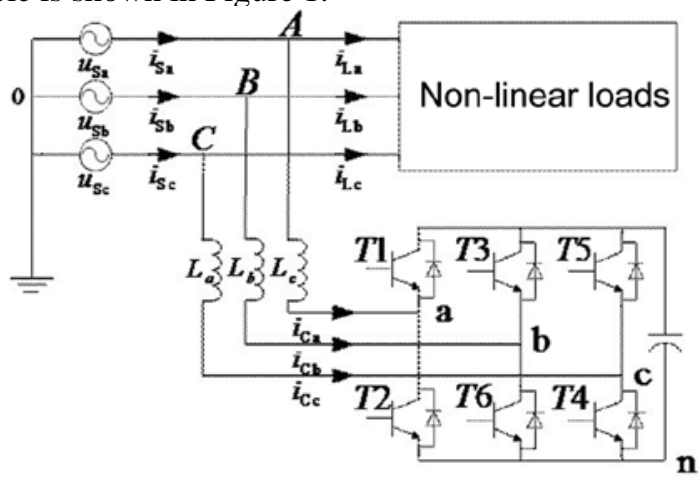

Figure 1. Circuit structure of the APF 
In the Figure $1, i_{S a}, i_{S b}, i_{S c}$ for the network side of the current; harmonic source for the various types of non-linear load; $i_{L a}, i_{L b}, i_{L c}$ for the harmonic and reactive components with the load current; $L_{a}, L_{b}, L_{c}$ for the connection inductance between the APF and the grid; $i_{C a}, i_{C b}, i_{C c}$ for APF issued by the compensation current, offset the harmonic and reactive power components ${ }^{[5]}$.

Set unipolar binary logic switching function:

$$
S_{K}=\left\{\begin{array}{ll}
1, & \text { Upper arm conduction } \\
0, & \text { Lower arm conduction }
\end{array} k=(a, b, c)\right.
$$

In the three-phase stationary coordinates, based on the Kirchhoff voltage law to write the system equation:

$$
\left\{\begin{array}{c}
L \frac{\mathrm{d} i_{\mathrm{Ca}}}{\mathrm{d} t}=u_{\mathrm{Sa}}-u_{\mathrm{c}} \mathrm{s}_{\mathrm{a}}-u_{\mathrm{n} 0} \\
L \frac{\mathrm{d} i_{\mathrm{Cb}}}{\mathrm{d} t}=u_{\mathrm{Sb}}-u_{\mathrm{c}} s_{\mathrm{b}}-u_{\mathrm{n} 0} \\
L \frac{\mathrm{d} i_{\mathrm{Cc}}}{\mathrm{d} t}=u_{\mathrm{Sc}}-u_{\mathrm{c}} s_{\mathrm{c}}-u_{\mathrm{n} 0} \\
C \frac{\mathrm{d} u_{\mathrm{c}}}{\mathrm{d} t}=i_{\mathrm{Ca}} s_{\mathrm{a}}+i_{\mathrm{Cb}} s_{\mathrm{b}}+i_{\mathrm{Cc}} s_{\mathrm{c}}
\end{array}\right.
$$

For a three-phase symmetric system:

$$
\left\{\begin{array}{c}
u_{\mathrm{Sa}}+u_{\mathrm{Sb}}+u_{\mathrm{Sc}}=0 \\
i_{\mathrm{Ca}}+i_{\mathrm{Cb}}+i_{\mathrm{Cc}}=0
\end{array}\right.
$$

(1) to (3) can be obtained:

$$
u_{\mathrm{n} 0}=-\frac{u_{\mathrm{c}}}{3} \sum_{\mathrm{k}=\mathrm{a}, \mathrm{b}, \mathrm{c}} \mathrm{S}_{\mathrm{k}}
$$

\section{SLIDING MODE CONTROLLER DESIGN}

The sliding mode variable structure controller with excellent performance consists of two basic steps : 1 ) Design a suitable sliding surface $S(x)$, resulting in a stable sliding mode dynamic; The control function $U(x)$ is designed to form the sliding mode region on the switching surface, which improves the motion quality of the normal approach segment. Once the functions $S(x)$ and $U(x)$ have been designed, a sliding mode variable structure control system can be established ${ }^{[6]}$. The active power filter generates the compensation current $i_{C}$ in real time by the command current $i_{C}{ }^{*}$, and suppresses the harmonic current in the network side current $i_{S}$ and compensates the reactive component. In actual operation, the compensation current $i_{C}$ can not track the instruction current $i_{C}{ }^{*}$ completely, and there is a deviation between them. The deviation is $\Delta i_{\mathrm{C}}=i_{\mathrm{C}}^{*}-i_{\mathrm{C}}$.

In the three-phase symmetric system, take $a$ phase for analysis. Let state variables:

$$
\left.\begin{array}{c}
x_{1}=i_{\mathrm{Ca}}^{*}-i_{\mathrm{Ca}} \\
x_{2}=x_{1}^{\prime}=-i_{\mathrm{Ca}}^{\prime}
\end{array}\right\}
$$

The equation of state is as follows:

$$
\left[\begin{array}{l}
\dot{x}_{1} \\
\dot{x}_{2}
\end{array}\right]=\left[\begin{array}{ll}
0 & 1 \\
0 & 0
\end{array}\right]\left[\begin{array}{l}
x_{1} \\
x_{2}
\end{array}\right]+\left[\begin{array}{l}
0 \\
-\mathrm{w}
\end{array}\right] u(x)
$$

In the formula: $U(x)$ is the control function; $\mathrm{W}$ is a constant, and $\mathrm{W}>0$. Set sliding surface:

$$
\mathrm{s}=\left[\begin{array}{ll}
\mathrm{v} & 1
\end{array}\right]\left[\begin{array}{l}
x_{1} \\
x_{2}
\end{array}\right]=\mathrm{v} x_{1}+x_{2}, \dot{s}=\left[\begin{array}{ll}
\mathrm{v} & 1
\end{array}\right]\left[\begin{array}{l}
\dot{x}_{1} \\
\dot{x_{2}}
\end{array}\right]=\mathrm{v} \dot{x_{1}+\dot{x}_{2}}
$$

In the formula: $\mathrm{v}$ is a constant.

Using proportional switching control law, A, B for the control parameters, which is:

By accessibility conditions:

$$
u_{\mathrm{a}}^{*}=\varphi(t)=A x_{1}+B x_{2}
$$

$$
s s^{\prime}=s\left[C x_{2}+\frac{1}{L}\left(A x_{1}+B x_{2}\right)\right]
$$

Deduced:

$$
A=\left\{\begin{array}{ll}
a_{1} & x_{1} s>0 \\
b_{1} & x_{1} s<0
\end{array} ; \quad B= \begin{cases}a_{2} & x_{2} s>0 \\
b_{2} & x_{2} s<0\end{cases}\right.
$$

It is deduced that the control function parameters should satisfy:

$$
\left.\begin{array}{c}
a_{1}>0, b_{1}<0 \\
a_{2}>c L, b_{2}<c L
\end{array}\right\}
$$

\section{OPTIMIZATION OF CONTROLLER PARAMETERS BY GENETIC ALGORITHM}

In the ideal case, the sliding mode control state point will slide along the sliding surface. In general modeling, the high frequency portion is ignored. The switch is easy to cause system jitter, and jitter may further excite high frequency dynamics that are ignored in modeling. High-frequency jitter will affect the sensor, resulting in damage to the system structure or control instability. In this paper, the genetic algorithm is used to optimize the parameters of the sliding mode controller. The theory is strong, which reduces the time taken to determine the parameters by the traditional trial and error method, improves the efficiency and improves the control effect.

\section{A. The Advantages of Genetic}

Compared with the traditional optimization methods, the genetic algorithm has the advantages of good convergence, less computation time and good robustness, and is widely used in engineering research. The algorithm has the advantages of large search space and strong global search ability, and can quickly obtain the global solution. The search process is not easy to fall into the trap of local optimal solution $^{[7]}$.

\section{B. The Construction and Improvement of Genetic Algorithm}

The individuals with large fitness function value will have more chance to generate new population and obtain the maximum solution of the function value. However, the optimization of the current controller parameter is to find the minimum value of the objective function ${ }^{[8]}$. Therefore, when the individual fitness is $\varphi$, the fitness function $\xi=1 / \varphi$ is constructed. In order to obtain the optimal dynamic 
characteristic, it is defined that the integral of current error absolute value to time is defined as the objective function, and the parameter is selected according to the minimum value of the objective function. Define the objective function:

$$
\varphi=\int_{0}^{\infty} t|e(t)| \mathrm{d} t
$$

In the formula: $e(t)$ is the system current error; $t$ is the simulation time.

In this paper, the fitness function is extended by the annealing algorithm, and the difference between the individuals is increased in the later period, which avoids the premature convergence and solves the problem of the local search ability of the genetic algorithm. The algorithm uses the following stretching methods:

$$
\begin{gathered}
\xi_{i}=\frac{\mathrm{e}^{\frac{\xi_{i}}{T}}}{\sum_{i=1}^{N} \mathrm{e}^{\frac{\xi_{i}}{T}}} \\
T=T_{0}\left(0.99^{\gamma-1}\right)
\end{gathered}
$$

In the formula: $\xi_{i}$ is the fitness of the $i$ th individual; $N$ is the population size; $\gamma$ is the genetic algebra; $T$ is the annealing temperature; $T_{0}$ is the initial annealing temperature.

Genetic algorithm can maintain the balance between search depth and search breadth. The selection mechanism is used to search the accumulated information in depth, and the genetic operator searches for new regions in the solution space.

For the selection mechanism, this paper combines the selection of competition with roulette selection, reproduces the chromosomes with high fitness, and then selects the chromosomes pairs by roulette, and brings the individuals with high fitness into the new population until new Population number. With the algorithm to run the number of individuals to ensure that the elite, while increasing the convergence rate can effectively prevent the trap into the local optimal algorithm ${ }^{[9]}$.

Genetic operators include crossover operators and mutation operators. The performance of the crossover operator largely determines the performance of the genetic system, and selects each character of the individual in the population as a potential crossing point ${ }^{[10]}$. The mutation operator is usually used as a minor operator, can improve the local search ability of genetic algorithm ${ }^{[11]}$.

\section{Controller Design Results}

Genetic algorithm generally find the maximum solution in the global, definition of fitness function $\varphi=-\gamma(x, y)$. The test function $\gamma(x, y)$ is simulated and the minimum value is calculated to verify the effectiveness of the genetic algorithm. The test functions are as follows:

$$
\gamma(x, y)=-30 \frac{\sin \sqrt{0.1+(x-5)^{2}+(y-5)^{2}}}{\sqrt{0.1+(x-5)^{2}+(y-5)^{2}}}
$$

Take 30 samples, in the formula 30 for generations of evolution, 0.8 for the crossover operator, 0.1 for the mutation operator.
As shown in Figure 3 simulation results, test function after 30 generations of genetic. The results of Fig.3 show that the minimum value of -25.6876 , which is the maximum fitness, is very close to the theoretical value of the global optimal solution after iteration of 30 generations of genetic algorithm, which shows that the constructed genetic algorithm is feasible.

The sliding mode controller is designed by genetic algorithm, and the result is as follows: $c=23.187, a_{1}=12.435$, $b_{1}=19.432, a_{2}=7.425, b_{2}=4.564$ 。

\section{EXPERIMENT ANALYSIS}

\section{A. System Simulation}

The simulation of three-phase parallel APF is carried out by Matlab/Simulink. The experimental conditions are: line voltage $380 \mathrm{~V}, \mathrm{f}=50 \mathrm{~Hz}$; AC side of the inductance $\mathrm{La}=\mathrm{Lb}$ $=\mathrm{Lc}=20 \mathrm{mH}$; take three-phase rectifier circuit for non-linear load, resistance $\mathrm{R}=15 \Omega$, inductance $\mathrm{L}=3 \mathrm{mH}$. The sliding mode controller based on genetic algorithm and the traditional PI control are experimentally verified under the condition of similar control parameters, and the dynamic response and steady state performance are analyzed. Taking phase A current as an example, the simulation load current waveform and spectrum analysis are shown in Fig. 3. The load current contains a large number of $6 k \pm 1$ order harmonics, and the total harmonic distortion (THD) is $28.83 \%$.

When the sliding mode control is used, the dynamic performance of the shunt active filter is obviously improved, and the compensation performance is good, the tracking performance is good and the current glitch is small. When the system is stable, the network-side current approaches the ideal sinusoidal waveform, and the sliding mode control has obvious advantages.

Figure 4 is the traditional PI control mode and genetic algorithm based on the sliding mode control after the compensation of the power supply current waveform, from the waveform can be seen in contrast, both control methods can achieve harmonic current compensation. In traditional PI control method, the chattering of the peak and the valley current is small, and the higher harmonic is large. The maximum amplitude of the chirp is $0.5 \mathrm{~A}$ at the peak, and the waveform of the power supply after compensation has obvious distortion. The maximum chattering amplitude of the sliding mode control method based on genetic algorithm is less than $0.2 \mathrm{~A}$, which effectively weakens the chattering and the sine of the current waveform is higher.

Figure 5 shows the two solutions after the power supply current spectrum analysis. The traditional PI control method THD is $4.58 \%$, and the optimized sliding mode control method THD is $2.36 \%$, which meets the national standard. But under the same conditions, the latter compensation current low harmonic content, can effectively reduce the distortion rate, the compensation effect is better. 

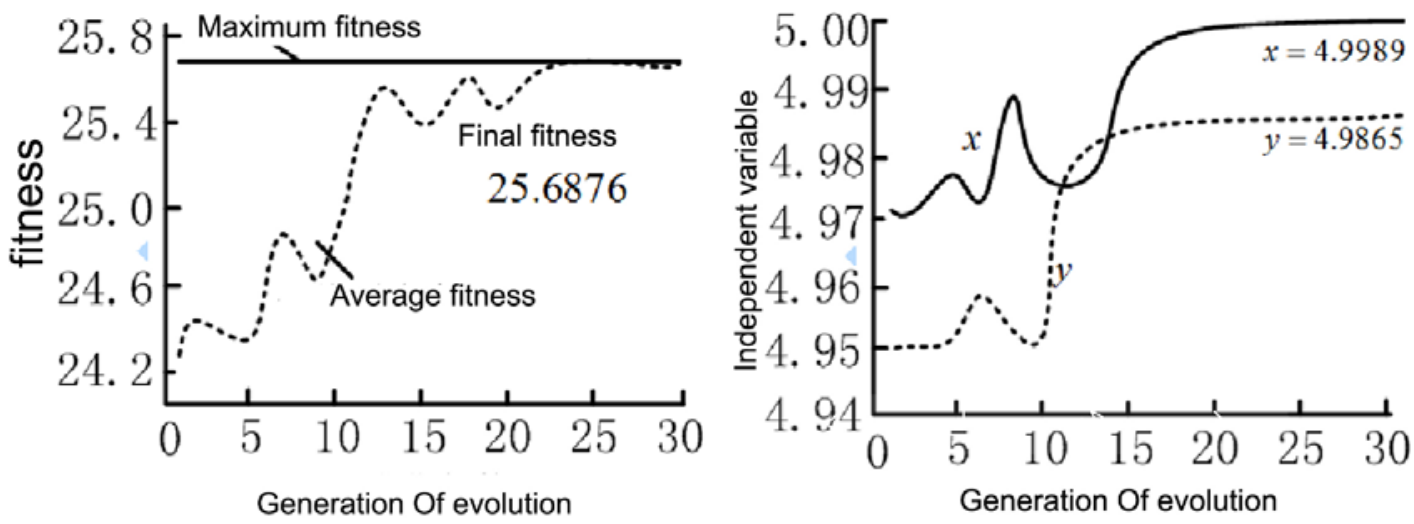

Figure 2. The test function of simulation results
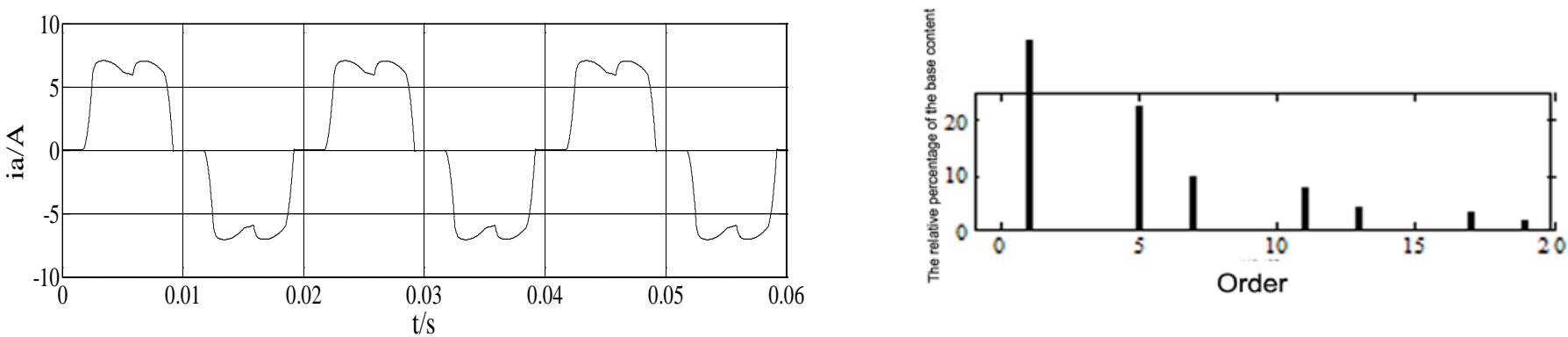

Figure 3. Simulation load current waveform and its analysis

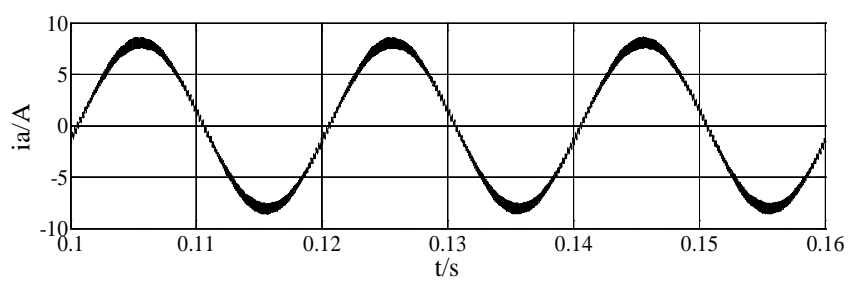

(a) Traditional PI control mode A-phase power supply current waveform

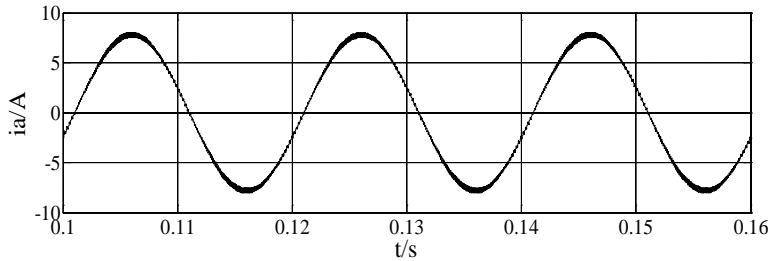

(b) Sliding mode control after optimization A-phase power supply current waveform

Figure 4. A-phase source current waveforms with the control of two methods

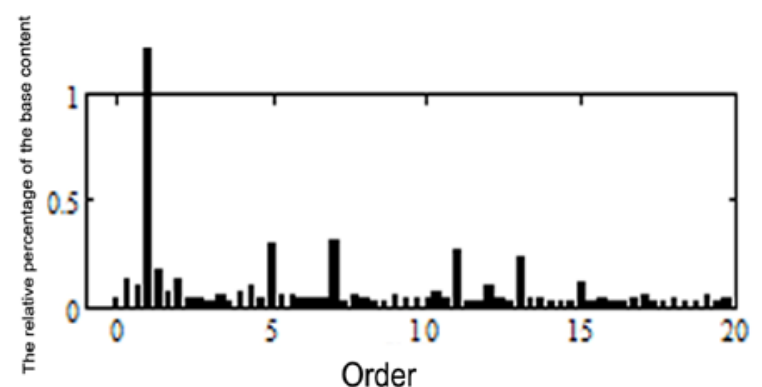

(a) Traditional PI control mode A-phase power supply current spectrum

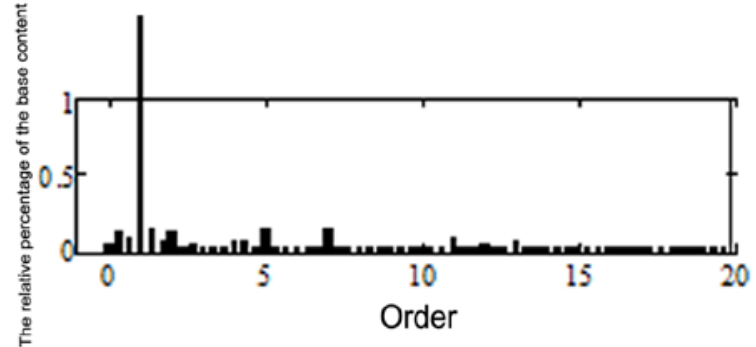

(b) Sliding mode control mode A - phase power supply current spectrum after optimization

Figure 5. A-phase source current spectrums under the control of two methods 
The optimized harmonic control is used to track and compensate the harmonic current. In the $0.1 \mathrm{~s}$ moment load mutation, the compensation after the power supply current as shown in Figure 6, the new control mode can also ensure a fast dynamic response in a cycle to reach stability.

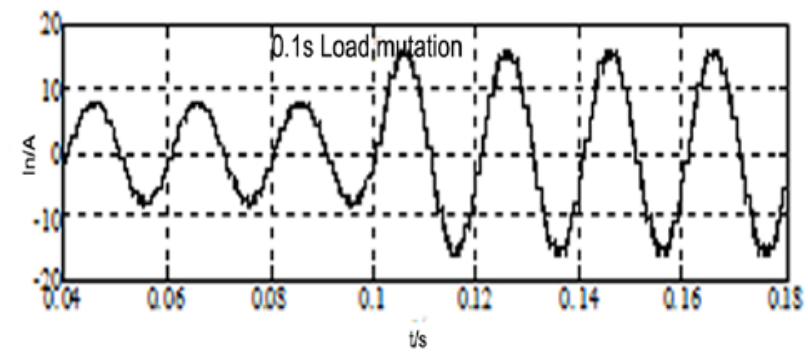

Figure 6. A-phase source current waveforms with the optimized control of sliding mode under load mutation.

\section{B. Experimental Verification}

In order to verify the design effect, the experimental lowvoltage three-phase shunt active power filter test bed is based on TMS320F28335 as the main control chip, the switching frequency is $10 \mathrm{kHz}$, the main parameters and simulation parameters are basically the same. Figure 7 shows the Aphase load current waveform and the A-phase power supply current waveform after optimization using sliding mode variable structure control with genetic algorithm. The compensated sine wave approximates the sine wave, which proves that the designed controller has better tracking compensation effect for harmonics ${ }^{[12]}$.

To verify the dynamic performance of the controller, the system is running during a sudden load change. As shown in Fig. 8, the result is the same as the simulation analysis. After a short period of fluctuation, the system enters a new steady state. The current does not appear in the transient process and the control effect is better.

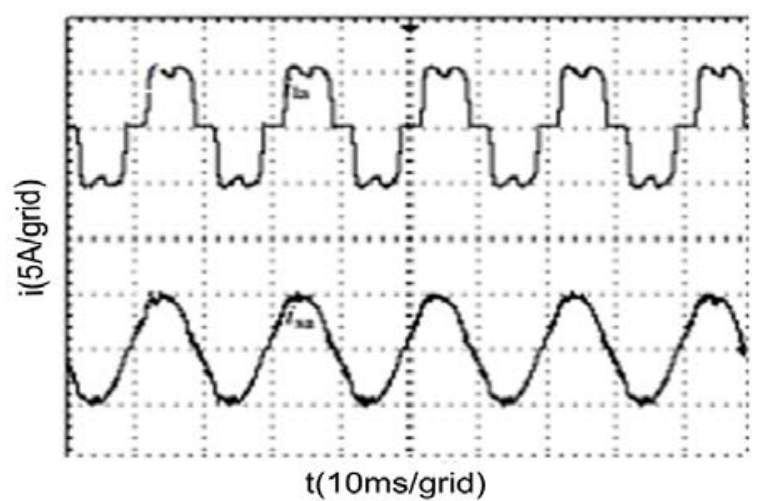

Figure 7. Waveforms of A-phase load current and A-phase source current after compensating

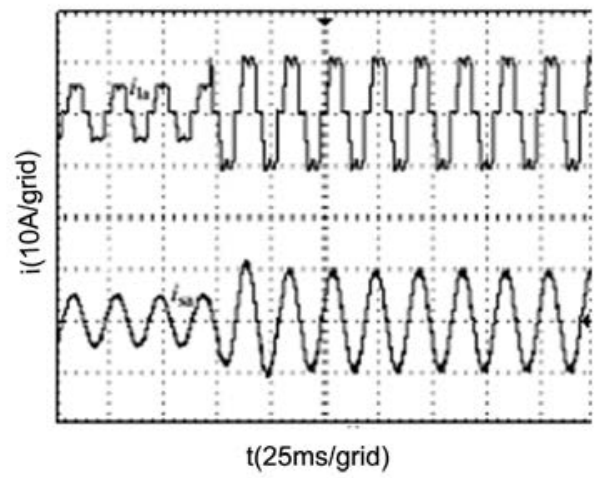

Figure 8. Waveforms of A-phasemutative load current and A-phase source current after compensating.

The experimental results show that the dynamic performance of shunt active power filter is improved obviously and the compensation performance is good. The tracking performance is good and the current glitch is small. When the system is stable, the net-side current approaches the ideal sinusoidal waveform.

\section{CONCLUSION}

In order to improve the active power filter jitter at high frequency, the system response time and anti-disturbance performance, this paper presents a sliding mode control based on genetic algorithm active power filter. Using genetic algorithm to optimize the parameters of the controller, the theory is strong, make up for the sliding mode control parameters try to take time-consuming flaws. The comparison between the traditional PI controller and the optimized sliding mode controller shows that the optimized sliding mode controller can meet the requirements of the fast and robustness of the active power filter. The proposed control strategy is feasible and has good theoretical and practical value.

\section{REFERENCES}

[1] LI Lan-fang,YANG Hong-geng,GUO Wei-ming. Stability control of the three-phase shunt active power filter based on the fuzzy control and Lyapunov theory [J].Power System Protection and Control, 2013,41(2): 150-155.

[2] GUO Ke,ZENG Yi,LIUQiang1,et al.Application of LCL-filter in photovoltaic grid-connected system with active power filter function [J]. Power System Protection and Control, 2013,41(3): 73-79.

[3] GAO Wei-bing. Variable structure control theory and design method[M]. Beijing: Science Publishing House,1988.

[4] QIAO Ming-zhong,XIA Yi-hui,LIANG Jing-hui,et al. Research on PI control based compound controller applying to shunt active filter[J].Power System Protection and Control,2013,41(14):54-59.

[5] LI Jing,ZHANG Xiao,LIU Xiaolei. Research on Sliding Mode Control Applied in DC-link Voltage Control for APF [J]. Power Electronics,2012,46(6): 39-41.

[6] CAI Liang-wei, LI Xia. Improvement on crossover operation of genetic algorithms[J]. Systems Engineering and Electronics, 2009, 28(6): 925-928.

[7] WU Zhong-qiang, ZHUANG Shu-yan, MA Bao-ming, et al. Research on adaptive fuzzy sliding mode control for grid-connected inverter based on inverse system[J]. Power System Protection and Control, 2011, 39(24): 1-7. 
[8] CHEN Zhong, LUO Ying-peng, SHI Lei, et al. Analysis on mechanisms of two typical control schemes for parallel APF[J]. Proceedings of the CSEE, 2010, 30(33): 37-43.on Power Systems, 2012, 27(2): 1-10.

[9] Kim Young-Jin, Ahn Seon-Ju, Hwang Pyeong-Ik, et al. Coordinated control of a DG and voltage control devices using a dynamic programming algorithm[J]. IEEE Trans.
[10] WANG Zhao-an, HUANG Jun. Power Electronies[M]. Beijing: Mechanieal Industrial Publishing House,2005.

[11] ZHANG Naiyao. Use Genetic Algorithem to Optimize Membership Function Parameters of fuzzy Controller[J]. Electric drive, 1996,18(1):4-6.

[12] YOU Li,TAO Jian,DAI Shaojun,et al.Research on the Active Power Filter Applied Into High-voltage System[J]. Power Electronics,2013,47(9):52-54. 